\title{
Multiwavelength variability and correlation studies of Mrk 421 during historically low X-ray and $\gamma$-ray activity in 2015-2016
}

\section{B. Banerjee,,$^{a, *}$ T. Terzić, ${ }^{b}$ D. Paneque ${ }^{c}$ and P. Majumdar ${ }^{d}$ on behalf of the MAGIC, FACT, and Fermi-LAT Collaboration ${ }^{\dagger}$}

${ }^{a}$ Gran Sasso Science Institute

Viale Francesco Crispi, 7, L'Aquila (AQ), I-67100

${ }^{b}$ University of Rijeka,

Department of Physics, 51000 Rijeka, Croatia

${ }^{c}$ Max-Planck-Institut für Physik,

D-80805 München, Germany

${ }^{d}$ Saha Institute of Nuclear Physics, HBNI, 1/AF Bidhannagar, Salt Lake, Sector-1, Kolkata 700064, India

E-mail: biswajit.banerjee@gssi.it

In this work, we report multi-band flux variability and correlations of the nearby $(\mathrm{z}=0.031)$ blazar Markarian 421 (Mrk 421) using multi-wavelength (MWL) data from November 2014 to June 2016. In this period, Mrk 421 exhibited historically low activity in X-rays and very-high-energy gamma rays (VHE; E $>0.1 \mathrm{TeV}$ ) and an additional spectral component was observed by Swift-BAT. The highest flux variability occurs in X-rays and VHE which, despite the low activity, show a significant positive correlation with no time lag. The hardness ratios in the X-rays and VHE $\gamma$-rays show the "harder-when-brighter" trend observed in many blazars. Interestingly, the trend flattens at the highest fluxes, which suggests different processes dominating the brightest states. Enlarging our data set with data from the years 2007 to 2014, we measured a positive correlation between the optical and $\mathrm{GeV}$ emission centered at zero time lag, and a positive correlation between the optical/GeV and the radio emission over a range of about 60 days centered at a time lag of $43+9 /-6$ days. This observation is consistent with the radio-bright zone being located about 0.2 parsec downstream from the optical/GeV emission regions. In most of the energy bands, the flux distribution follows the Lognormal, rather than the Normal function, indicating that the variability may be dominated by a multiplicative process.

$37^{\text {th }}$ International Cosmic Ray Conference (ICRC 2021)

July 12th - 23rd, 2021

Online - Berlin, Germany

\footnotetext{
${ }^{*}$ Presenter

$\dagger$ a complete list of authors can be found at the end of the proceedings
} 


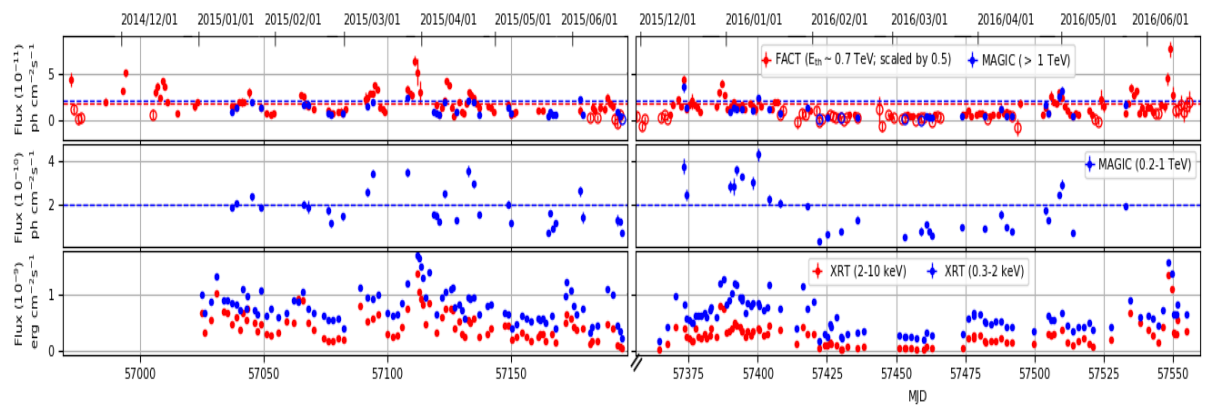

Figure 1: The VHE $\gamma$-ray (MAGIC \& FACT) and X-ray light curves (Swift-XRT) during 2015-2016 campaign. Reprinted from [3] (Monthly Notices of the Royal Astronomical Society, Volume 504, Issue 1, pp.1427-1451), Fig. 2.

\section{Introduction}

Blazars are subclass of active galactic nuclei (AGNs) with relativistic jets pointing towards the observer. Markarian 421 (Mrk 421) is a bright blazar located at redshift (z) of 0.031. The spectral energy distribution (SED) of blazars shows a double hump structure, where the low and high energy bump appears in the $\mathrm{keV}$ and $\mathrm{GeV}$ band, respectively. The origin of the first hump is commonly associated with the synchroton emission of the electrons inside the jet of the blazar. The high-energy bump is widely believed to be due to the inverse Compton emission of the synchrotron photons by the same population of electrons which produced them (synchrotron self Compton process; SSC $[8,17]$ ) or by Compton emission of the external thermal photons outside the jet (external Compton process; EC). Mrk 421 is a very well studied blazar showing variability across the entire electomagnetic band from radio to VHE $\gamma$-rays. The source has been studied extensively during outbursts in the past. However, less effort has been expended to explain the behaviour of the source during the low flux states. During the coordinated multiwavelegth (MWL) observations in 2015-2016, the source was found in a very low flux state during a two month period in 2016. This data set during 2015-2016 contains simultaneous (up to $2 \mathrm{hrs}$ for a single night) X-ray and VHE $\gamma$-ray observations. This enables us to study accurately the multiband variability and correlation during very low blazar activity. In order to establish some of the interesting results for correlation and the study of the flux distribution, we used data from previous MWL campaigns related to previous years back to 2007 [4-7]. Figure 1 shows the X-ray (0.3-2 keV and 2-10 keV) and VHE $\gamma$-ray (MAGIC and FACT) light curves (LC) of Mrk 421 during 2015-2016. The details of this study are published in [3]. Please refer to [3] if you want to cite this work.

\section{Appearance of a new component at hard X-ray energies}

The synchrotron hump of Mrk 421 consisting of optical, UV, and X-rays (Swift-XRT and -BAT) has been fitted with a log-parabola function. During MJD 57422-57429, Mrk 421 showed a very low X-ray flux and soft X-ray spectra while the flux in the $15-50 \mathrm{keV}$ band from Swift-BAT showed non-agreement with the log-parabola model (see Fig. 2). This is the first time that BAT measures a flux significantly above the extrapolated XRT spectral data. An excess in the hard X-rays has been previously reported by [12] for Mrk 421, using NuSTAR data in 2013. The BAT excess in this case 


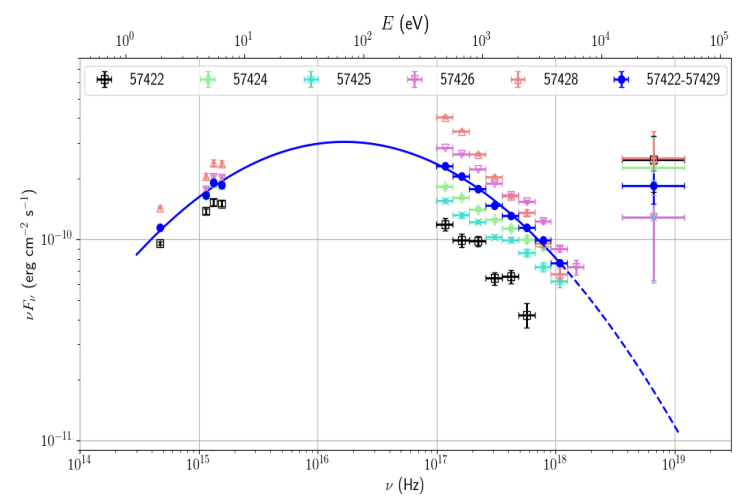

Figure 2: Log-parabola fit to the synchrotron spectrum of Mrk 421 during MJD 57422-57429. Reprinted from [3] (Monthly Notices of the Royal Astronomical Society, Volume 504, Issue 1, pp.1427-1451), Fig. 9.

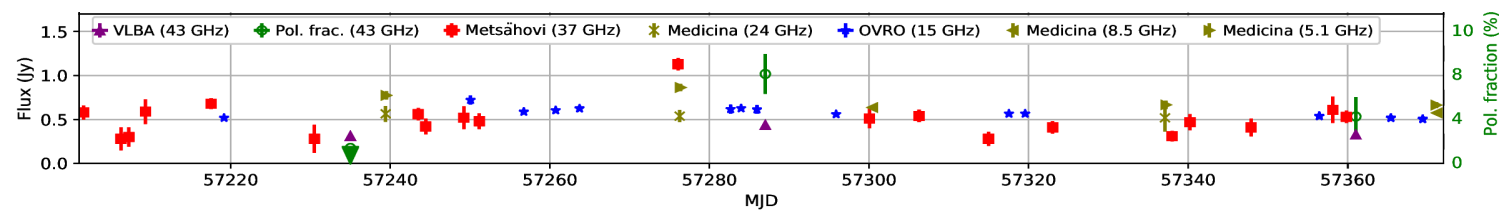

Figure 3: Radio flare detected by Metsähovi (37 GHz). Reprinted from [3] (Monthly Notices of the Royal Astronomical Society, Volume 504, Issue 1, pp.1427-1451), Fig. 3.

of Mrk 421 during 2015-2016 can be related to the presence of an occasionally appearing narrow spectral component which is very similar to the one reported for Mrk 501 at the TeV energies [13] or the onset of the IC component [10]. The details of this study are published in [3]. Please refer to [3] if you want to cite this work.

\section{Radio flare}

We report, for the first time, a radio flare observed by the Metsähovi telescope (at $37 \mathrm{GHz}$ ) on 2015 September 11, where a flux increased by a factor of two with a temporal timescale shorter than 3 weeks. However, the flux densities observed with Medicina (at $5 \mathrm{GHz}$ and $24 \mathrm{GHz}$ ) on September 11 , show an enhancement at $5 \mathrm{GHz}$ only and no changes at the $24 \mathrm{GHz}$ band. The VLBA observation performed on September 22 shows an increase in the polarization fraction which comes back to the normal values on December 5 . This increase in flux and a decrease in polarization in the radio band at $37 \mathrm{GHz}$ can be explained by a momentary disruption of the ordering of the magnetic field followed by an acceleration of the particles via a kink instability. The details of this study are published in [3]. Please refer to [3] if you want to cite this work.

\section{Variability and correlation studies}

Mrk 421 showed a very low activity in the X-ray and VHE $\gamma$-rays during 2015-2016. The hardness ratio (ratio of the flux in hard band flux to the soft band flux) in the $\mathrm{keV}$ and $\mathrm{TeV}$ band given by $\mathrm{HR}_{\mathrm{keV}}$ and $\mathrm{HR}_{\mathrm{TeV}}$, respectively, also showed periods of extreme softness particularly around the period of MJD 57422 to MJD 57474. This is for the first time, a flattening of the in the HR vs. flux 


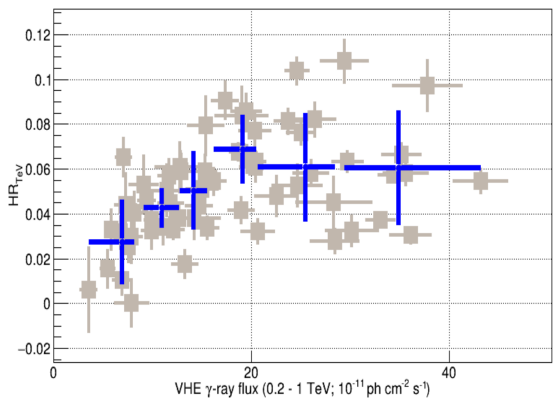

Figure 4: Flattening of HR in the VHE $\gamma$-ray band $\left(\mathrm{HR}_{\mathrm{TeV}}=\frac{\text { Flux }_{>0.1 \mathrm{TeV}}}{\text { Flux }_{0.2-1 \mathrm{TeV}}}\right)$. Reprinted from [3] (Monthly Notices of the Royal Astronomical Society, Volume 504, Issue 1, pp.1427-1451), Fig. 7.

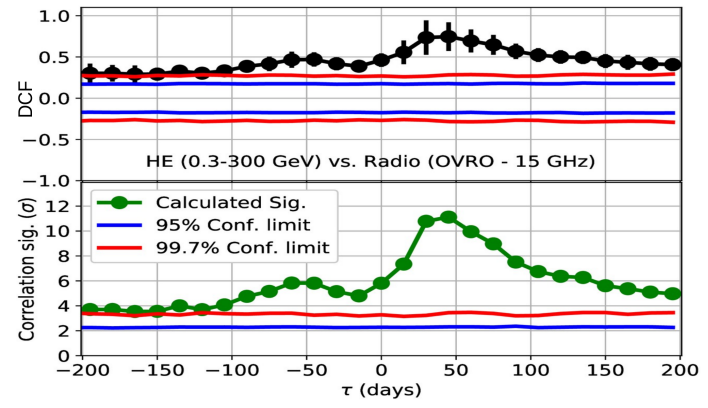

Figure 5: Correlation between the radio (OVRO; $15 \mathrm{GHz}$ ) and the HE $\gamma$-rays, which peaks at around 45 days. Reprinted from [3] (Monthly Notices of the Royal Astronomical Society, Volume 504, Issue 1, pp.1427-1451), Fig. 12.
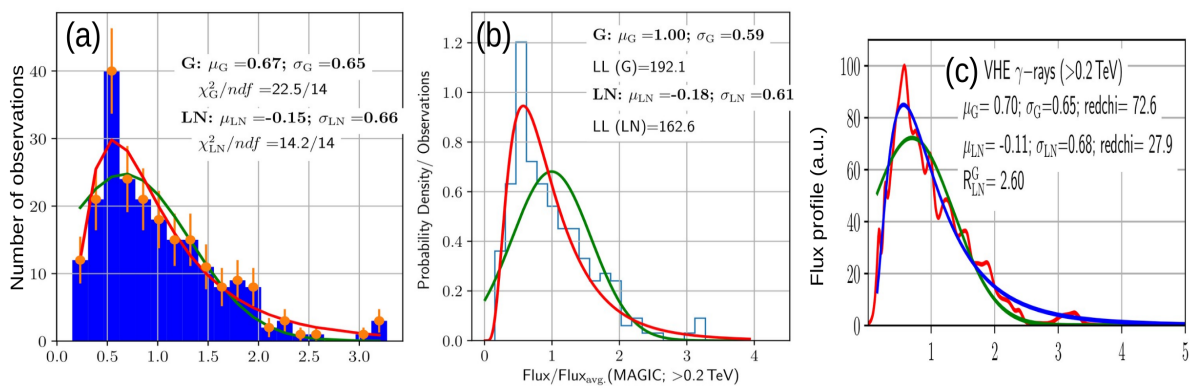

Figure 6: Flux distribution of Mrk 421 in the VHE $\gamma$-rays using: (a) $\chi^{2}$ minimization, (b) Log-likelihood, and (c) Flux profile methods. Reprinted from [3] (Monthly Notices of the Royal Astronomical Society, Volume 504, Issue 1, pp.1427-1451), Fig. 13, E2, and G2.

trend has been seen (see Figure 4) in the VHE $\gamma$-rays similar to the studies in the X-rays [7]. The highest variability quantified in terms of the fractional variability is observed in the highest X-ray and VHE $\gamma$-ray energies at a similar level.

Correlated behaviour between the LCs is quantified using Pearson and Discrete Correlation function (DCF, [11]) and evaluated the significance with Monte Carlo simulations, see [3] for details. The strongly correlated zero-lag behaviour between the VHE and X-ray emissions, persistent during the 2015-2016 observing campaigns, indicates that the X-ray and VHE $\gamma$-ray emissions are dominated by leptonic scenarios (presumably SSC), where the same population of high-energy electrons radiate simultaneously at X-ray and VHE. A significant $(>3 \sigma)$ correlated behaviour, between the $>2 \mathrm{GeV}$ emission measured with Fermi-LAT and the VHE fluxes measured with FACT $\left(\mathrm{E}_{t h} \sim 0.7 \mathrm{TeV}\right)$ has been observed quantified in time steps of \pm 3 days only for time lag $\tau=0$, indicating that the emission in these two energy bands is simultaneous within the resolution of the study. We found a positive correlation between the $>0.3 \mathrm{GeV}$ emission (from Fermi-LAT) and optical (R-band) emission for a range of about 60 days centered at $\tau=0$, which confirms the results of [9]. This indicates that these two bands, belonging to the rising segments of the two SED bumps (and located somewhat close to the peak of the bumps) may indeed be produced (at least partially) by the same particle population and in the same region (or regions). A positive correlation has 
been found between the $>0.3 \mathrm{GeV}$ emission (from Fermi-LAT) and the radio emission at $15 \mathrm{GHz}$ and $37 \mathrm{GHz}$ (from OVRO and Metsähovi) for a range of about 60 days centered at $\tau \sim 45$ days. This indicates that the radio emission occurs about 45 days after the $\mathrm{GeV}$ emission. The same correlation with the same time lag occurs also for the optical and the radio emissions. Figure 5 shows the correlation between HE $\gamma$-ray and OVRO LCs. The details of this study are published in [3]. Please refer to [3] if you want to cite this work.

\section{Determination of the flux distribution using the flux profile method}

Using the data from 2007 to $2016^{1}$, we quantify the flux variations with a method largely inspired by the kernel density estimation (KDE), dubbed "flux profile construction" that allows us to estimate the flux distributions even for flux measurements with relatively large errors.

We treat each flux measurement from a LC as a Gaussian with the flux values as the mean and the flux uncertainty as the standard deviation. The amplitude is inversely proportional to the standard deviation, so that the area under each individual Gaussian is unity. A "flux profile" for a LC is constructed by adding individual flux measurements. We determine the preferred shape of a flux profile, by fitting the Gaussian and LogNormal functions as mentioned in [3]. Using this methodology, we determined the most probable flux values and the dispersion in the flux values for all the bands probed. Figure 6 shows the flux distribution in the VHE $\gamma$-rays using three different methods (see [3] for details): a) $\chi^{2}$ minimization, b) loglikelihood, and c) flux-profile.

A LogNormal distribution of flux may imply that the emission is being powered by a multiplicative process rather than an additive one which may be resulted from the fluctuations in the accretion disk $[15,16]$. Signature of lognormality in blazars may lead to the conclusion that the variability in flux may be originated in the accretion disks and not from the jet. The details of this study are published in [3]. Please refer to [3] if you want to cite this work.

\section{Summary}

We observed a historically low flux state in the X-rays on MJD 57364 (integrated flux in the $2-10 \mathrm{keV}$ band with a flux of $\left.(2.41 \pm 0.15) \times 10^{-11} \mathrm{erg} \mathrm{cm}^{-2} \mathrm{~s}^{-1}\right)$. During the 2015-2016 campaign, MAGIC observed the lowest flux state in the $0.2-1 \mathrm{TeV}$ with flux of $(3.56 \pm 0.91) \times 10^{-11} \mathrm{ph} \mathrm{cm}^{-2} \mathrm{~s}^{-1}$, on MJD 57422. During the 7 day interval, MJD 57422-57429, we detected emission at high flux at hard X-ray $(15-50 \mathrm{keV})$ with Swift-BAT which is higher than the extrapolated one from the XRT spectra $(0.3-10 \mathrm{keV})$. This is the first time that this excess Swift-BAT $(15-50 \mathrm{keV})$ emission has been observed from Mrk 421. Its origin may be interpreted as the presence of an occasionally appearing narrow spectral component (as seen in Mrk 501; [13]), or may be produced by the onset of the SSC component [12]. A detailed explanation will be presented in a follow-up paper. Metsähovi (37 GHz) observed a radio flare on 2015 September 11 with a flux doubling timescale below 21 days. This is the first time that such a rapid change in flux was seen in the radio band. Although, no change in the flux was observed in the $24 \mathrm{GHz}$ band by Medicina, enhanced activity at $5 \mathrm{GHz}$ (also with Medicina) was observed. We noted an increase in the polarization fraction with VLBA

\footnotetext{
${ }^{1}$ The two large VHE $\gamma$-ray flaring episodes of Mrk 421 in 2010 February [1] and 2013 April [2] have been excluded to avoid large biases in the distributions.
} 
on September 22. This increase in flux and polarization may be caused by a momentary disruption of the ordering of the magnetic field followed by a particle acceleration via a kink instability. Our study revealed a flattening of the hardness ratio with the increase in flux, which is more pronounced in the soft band of VHE. We observed the correlation between the X-ray and VHE $\gamma$-rays with more than $5 \sigma$ even in the low flux states during 2015-2016. Our study suggests a correlation between the optical/HE gamma rays and radio emission with a time delay of around 45 days. This observation is consistent with the radio-bright zone being located about 0.2 parsec downstream from the optical/GeV emission regions [14]. We report a correlation between the VHE $\gamma$-rays (observed with FACT with $\mathrm{E}_{t h} \sim 0.7 \mathrm{TeV}$ ) vs. HE gamma rays in the energy band 2-300 GeV. However, no significant correlation between the VHE and HE gamma rays in the $0.2-2 \mathrm{GeV}$ has been found. This may be an indication of the presence of two gamma-ray emitting regions. The shape of the MWL flux distributions can shed light on the origin of the blazar variability. In this work, we devised two methods to estimate the flux distribution in a way that is less biased by the flux binning and that takes the flux uncertainties into account. Most of the MWL flux distributions are consistent with a lognormal distribution, which suggests that the variability is caused by a multiplicative process. The details of this study are published in [3]. Please refer to [3] if you want to cite this work.

\section{References}

[1] Abeysekara A. U., et al., 2020, ApJ, 890, 97

[2] Acciari V. A., et al., 2020, ApJS, 248, 29

[3] Acciari et al. V. A., 2021, MNRAS, 504, 1427

[4] Ahnen M. L., et al., 2016, A\&A, 593, A91

[5] Aleksić J., et al., 2012, A\&A, 542, A100

[6] Aleksić J., et al., 2015, A\&A, 578, A22

[7] Baloković M., et al., 2016, ApJ, 819, 156

[8] Bloom S. D., Marscher A. P., 1996, ApJ, 461, 657

[9] Carnerero M. I., et al., 2017, MNRAS, 472, 3789

[10] Chen L., 2017, ApJ, 842, 129

[11] Edelson R. A., Krolik J. H., 1988, ApJ, 333, 646

[12] Kataoka J., Stawarz Ł., 2016, ApJ, 827, 55

[13] MAGIC Collaboration et al., 2020, A\&A, 637, A86

[14] Max-Moerbeck W., et al., 2014, MNRAS, 445, 428

[15] McHardy I., 2010, X-Ray Variability of AGN and Relationship to Galactic Black Hole Binary Systems. p. 203, doi:10.1007/978-3-540-76937-8_8 
[16] Uttley P., McHardy I. M., Vaughan S., 2005, MNRAS, 359, 345

[17] van den Berg J. P., Böttcher M., Domínguez A., López-Moya M., 2019, ApJ, 874, 47

\section{Acknowledgments}

The MAGIC collaboration acknowledges the support from the agencies and organizations listed here: https://magic.mpp.mpg.de/acknowledgments_ICRC2021.

The Fermi-LAT Collaboration acknowledges generous ongoing support from a number of agencies and institutes that have supported both the development and the operation of the LAT as well as scientific data analysis. These include the National Aeronautics and Space Administration and the Department of Energy in the United States, the Commissariat á l'Energie Atomique and the Centre National de la Recherche Scientifique / Institut National de Physique Nucléaire et de Physique des Particules in France, the Agenzia Spaziale Italiana and the Istituto Nazionale di Fisica Nucleare in Italy, the Ministry of Education, Culture, Sports, Science and Technology (MEXT), High Energy Accelerator Research Organization (KEK) and Japan Aerospace Exploration Agency (JAXA) in Japan, and the K. A. Wallenberg Foundation, the Swedish Research Council and the Swedish National Space Board in Sweden.

Additional support for science analysis during the operations phase is gratefully acknowledged from the Istituto Nazionale di Astrofisica in Italy and the Centre National d'Étude Spatiales in France. This work performed in part under DOE Contract DE-AC02-76SF00515.

BB acknowledges financial support from MIUR (PRIN 2017 grant 20179ZF5KS).

\section{Complete list of authors}

The MAGIC Collaboration: V. A. Acciari ${ }^{1}$, S. Ansoldi ${ }^{2,41}$, L. A. Antonelli ${ }^{3}$, M. Artero ${ }^{5}$, K. Asano ${ }^{6}$, A. Babic ${ }^{8}$, B. Banerjee ${ }^{7}$ A. Baquero ${ }^{9}$, U. Barres de Almeida ${ }^{10}$, J. A. Barrio ${ }^{9}$, I. Batkovic ${ }^{11}$, J. Becerra González ${ }^{1}$, W. Bednarek ${ }^{12}$, L. Bellizzi ${ }^{13}$, E. Bernardini ${ }^{14}$, M. Bernardos ${ }^{11}$, A. Berti ${ }^{15}$, J. Besenrieder ${ }^{15}$, W. Bhattacharyya ${ }^{14}$, C. Bigongiari ${ }^{3}$, O. Blanch ${ }^{5}$, H. Bökenkamp ${ }^{7}$, G. Bonnoli ${ }^{16}$, Ž. Bošnjak ${ }^{8}$, G. Busetto ${ }^{11}$, R. Carosii ${ }^{17}$, G. Ceribella ${ }^{15}$, M. Cerruti ${ }^{18}$, Y. Chai ${ }^{15}$, A. Chilingarian ${ }^{19}$, S. Cikota ${ }^{8}$, S. M. Colak ${ }^{5}$, E. Colombo ${ }^{1}$, J. L. Contreras ${ }^{9}$, J. Cortina ${ }^{20}$, S. Covino ${ }^{3}$, G. D’Amico ${ }^{15,42}$, V. D’Elia ${ }^{3}$, P. Da Vela ${ }^{17,43}$, F. Dazzi ${ }^{3}$, A. De Angelis ${ }^{11}$, B. De Lotto ${ }^{2}$, M. Delfino ${ }^{5,44}$, J. Delgado ${ }^{5,44}$, C. Delgado Mendez ${ }^{20}$, D. Depaoli ${ }^{21}$, F. Di Pierro ${ }^{21}$, L. Di Venere ${ }^{22}$, E. Do Souto Espiñeira ${ }^{5}$, D. Dominis Prester ${ }^{23}$, A. Donini ${ }^{2}$, M. Doro ${ }^{11}$, V. Fallah Ramazani ${ }^{25,45}$, A. Fattorini ${ }^{7}$, M. V. Fonseca ${ }^{9}$, L. Font ${ }^{26}$, C. Fruck ${ }^{15}$, S. Fukami ${ }^{6}$, Y. Fukazawa ${ }^{27}$, R. J. García López ${ }^{1}$, M. Garczarczyk ${ }^{14}$, S. Gasparyan ${ }^{28}$, M. Gaug ${ }^{26}$, N. Giglietto ${ }^{22}$, F. Giordano ${ }^{22}$, P. Gliwny ${ }^{12}$, N. Godinović ${ }^{29}$, J. G. Green ${ }^{3}$, D. Green ${ }^{15}$, D. Hadasch ${ }^{6}$, A. Hahn ${ }^{15}$, L. Heckmann ${ }^{15}$, J. Herrera ${ }^{1}$, J. Hoang ${ }^{9,46}$, D. Hrupec ${ }^{30}$, M. Hütten ${ }^{15}$, T. Inada $^{6}$, K. Ishio ${ }^{12}$, Y. Iwamura ${ }^{6}$, I. Jiménez Martínez ${ }^{20}$, J. Jormanainen ${ }^{25}$, L. Jouvin ${ }^{5}$, M. Karjalainen ${ }^{1}$, D. Kerszberg ${ }^{5}$, Y. Kobayashi ${ }^{6}$, H. Kubo ${ }^{31}$, J. Kushida ${ }^{32}$, A. Lamastra ${ }^{3}$, D. Lelas ${ }^{29}$, F. Leone ${ }^{3}$, E. Lindfors ${ }^{25}$, L. Linhoff ${ }^{7}$, S. Lombardi ${ }^{3}$, F. Longo ${ }^{2,47}$, R. López-Coto ${ }^{11}$, M. López-Moya ${ }^{9}$, A. López-Oramas ${ }^{1}$, S. Loporchio ${ }^{22}$, B. Machado de Oliveira Fraga ${ }^{10}$, C. Maggio ${ }^{26}$, P. Majumdar ${ }^{33}$, M. Makariev $^{34}$, M. Mallamaci ${ }^{11}$, G. Maneva ${ }^{34}$, M. Manganaro ${ }^{23}$, L. Maraschi ${ }^{3}$, M. Mariotti ${ }^{11}$, M. Martínez $^{5}$, D. Mazin ${ }^{6,15}$, S. Menchiari ${ }^{13}$, S. Mender $^{7}$, S. Mićanović2 ${ }^{23}$, D. Miceli ${ }^{2,49}$, T. Miener ${ }^{9}$, J. M. Miranda ${ }^{13}$, R. Mirzoyan ${ }^{15}$, E. Molina ${ }^{18}$, A. Moralejo ${ }^{5}$, D. Morcuende ${ }^{9}$, V. Moreno ${ }^{26}$,

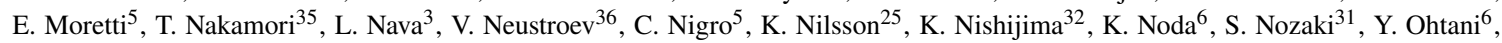
T. Oka ${ }^{31}$, J. Otero-Santos ${ }^{1}$, S. Paiano ${ }^{3}$, M. Palatiello ${ }^{2}$, D. Paneque ${ }^{15}$, R. Paoletti ${ }^{13}$, J. M. Paredes ${ }^{18}$, L. Pavletić ${ }^{23}$, P. Peñil ${ }^{9}$, M. Persic ${ }^{2,50}$, M. Pihet ${ }^{15}$, P. G. Prada Moroni ${ }^{17}$, E. Prandini ${ }^{11}$, C. Priyadarshi ${ }^{5}$, I. Puljak ${ }^{29}$, M. Ribó ${ }^{18}$, J. Rico ${ }^{5}$, C. Righi ${ }^{3}$, A. Rugliancich ${ }^{17}$, N. Sahakyan ${ }^{28}$, T. Saito ${ }^{6}$, S. Sakurai ${ }^{6}$, K. Satalecka ${ }^{14}$, F. G. Saturni ${ }^{3}$, B. Schleicher ${ }^{24}$, K. Schmidt ${ }^{7}$, T. Schweizer ${ }^{15}$, J. Sitarek ${ }^{12}$, I. Šnidarićc ${ }^{37}$, D. Sobczynska ${ }^{12}$, A. Spolon ${ }^{11}$, A. Stamerra ${ }^{3}$, J. Strišković ${ }^{30}$, D. Strom ${ }^{15}$, M. Strzys ${ }^{6}$, Y. Suda ${ }^{27}$, T. Suric ${ }^{37}$, M. Takahashi ${ }^{6}$,

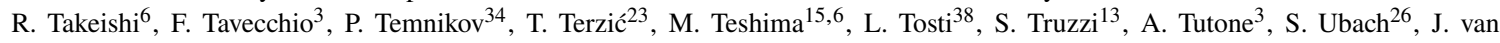
Scherpenberg ${ }^{15}$, G. Vanzo ${ }^{1}$, M. Vazquez Acosta ${ }^{1}$, S. Ventura ${ }^{13}$, V. Verguilov ${ }^{34}$, C. F. Vigorito ${ }^{21}$, V. Vitale ${ }^{39}$, I. Vovk ${ }^{6}$, M. Will ${ }^{15}$, C. Wunderlich ${ }^{13}$, T. Yamamoto ${ }^{40}$, and D. Zaric ${ }^{29}$

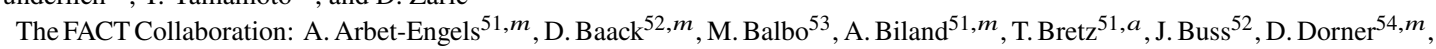
L. Eisenberger ${ }^{54}$, D. Elsaesser ${ }^{52, m}$, D. Hildebrand ${ }^{51}$, R. Iotov ${ }^{52}$, A. Kalenski ${ }^{54}$, K. Mannheim ${ }^{54, m}$, A. Mitchell ${ }^{51}$, D. Neise ${ }^{51}$, M. Noethe ${ }^{52}$, A. Paravac ${ }^{54}$, W. Rhode ${ }^{52, m}$, B. Schleicher ${ }^{54, m}$, V. Sliusar ${ }^{53}$, R. Walter ${ }^{53}$, 
The MWL collaborators: J. Valverde ${ }^{55}$, D. Horan $^{55}$, M. Giroletti ${ }^{57}$, M. Perri ${ }^{58,59}$, F. Verrecchia ${ }^{58,59}$, C. Leto ${ }^{58,60}$, A. C. Sadun ${ }^{61}$, J. W. Moody ${ }^{62}$, M. Joner ${ }^{62}$, A. P. Marscher ${ }^{63}$, S. G. Jorstad ${ }^{63,64}$, A. Lähteenmäki ${ }^{65,66}$, M. Tornikoski ${ }^{65}$, V. Ramakrishnan ${ }^{65,67}$, E. Järvelä ${ }^{65,68}$, R. J. C. Vera ${ }^{65,66}$, S. Righini ${ }^{69}$, and A.Y. Lien ${ }^{70,71}$

${ }^{1}$ Instituto de Astrofísica de Canarias and Dpto. de Astrofísica, Universidad de La Laguna, E-38200, La Laguna, Tenerife, Spain ${ }^{2}$ Università di Udine and INFN Trieste, I-33100 Udine, Italy ${ }^{3}$ National Institute for Astrophysics (INAF), I-00136 Rome, Italy ${ }^{5}$ Institut de Física d'Altes Energies (IFAE), The Barcelona Institute of Science and Technology (BIST), E-08193 Bellaterra (Barcelona), Spain ${ }^{6}$ Japanese MAGIC Group: Institute for Cosmic Ray Research (ICRR), The University of Tokyo, Kashiwa, 277-8582 Chiba, Japan ${ }^{7}$ Gran Sasso Science Institute, Viale Francesco Crispi, 7, L'Aquila (AQ), I-67100 ${ }^{8}$ Croatian MAGIC Group: University of Zagreb, Faculty of Electrical Engineering and Computing (FER), 10000 Zagreb, Croatia ${ }^{9}$ IPARCOS Institute and EMFTEL Department, Universidad Complutense de Madrid, E-28040 Madrid, Spain ${ }^{10}$ Centro Brasileiro de Pesquisas Físicas (CBPF), 22290-180 URCA, Rio de Janeiro (RJ), Brazil ${ }^{11}$ Università di Padova and INFN, I-35131 Padova, Italy ${ }^{12}$ University of Lodz, Faculty of Physics and Applied Informatics, Department of Astrophysics, 90-236 Lodz, Poland ${ }^{13}$ Università di Siena and INFN Pisa, I-53100 Siena, Italy ${ }^{14}$ Deutsches Elektronen-Synchrotron (DESY), D-15738 Zeuthen, Germany ${ }^{15}$ Max-Planck-Institut für Physik, D-80805 München, Germany ${ }^{16}$ Instituto de Astrofísica de Andalucía-CSIC, Glorieta de la Astronomía s/n, 18008, Granada, Spain ${ }^{17}$ Università di Pisa and INFN Pisa, I-56126 Pisa, Italy ${ }^{18}$ Universitat de Barcelona, ICCUB, IEEC-UB, E-08028 Barcelona, Spain ${ }^{19}$ Armenian MAGIC Group: A. Alikhanyan National Science Laboratory, 0036 Yerevan, Armenia ${ }^{20}$ Centro de Investigaciones Energéticas, Medioambientales y Tecnológicas, E-28040 Madrid, Spain ${ }^{21}$ INFN MAGIC Group: INFN Sezione di Torino and Università degli Studi di Torino, I-10125 Torino, Italy ${ }^{22}$ INFN MAGIC Group: INFN Sezione di Bari and Dipartimento Interateneo di Fisica dell’Università e del Politecnico di Bari, I-70125 Bari, Italy ${ }^{23}$ Croatian MAGIC Group: University of Rijeka, Department of Physics, 51000 Rijeka, Croatia ${ }^{25}$ Finnish MAGIC Group: Finnish Centre for Astronomy with ESO, University of Turku, FI-20014 Turku, Finland ${ }^{26}$ Departament de Física, and CERES-IEEC, Universitat Autònoma de Barcelona, E-08193 Bellaterra, Spain ${ }^{27}$ Japanese MAGIC Group: Physics Program, Graduate School of Advanced Science and Engineering, Hiroshima University, 739-8526 Hiroshima, Japan ${ }^{28}$ Armenian MAGIC Group: ICRANet-Armenia at NAS RA, 0019 Yerevan, Armenia ${ }^{29}$ Croatian MAGIC Group: University of Split, Faculty of Electrical Engineering, Mechanical Engineering and Naval Architecture (FESB), 21000 Split, Croatia ${ }^{30}$ Croatian MAGIC Group: Josip Juraj Strossmayer University of Osijek, Department of Physics, 31000 Osijek, Croatia ${ }^{31}$ Japanese MAGIC Group: Department of Physics, Kyoto University, 606-8502 Kyoto, Japan ${ }^{32}$ Japanese MAGIC Group: Department of Physics, Tokai University, Hiratsuka, 259-1292 Kanagawa, Japan ${ }^{33}$ Saha Institute of Nuclear Physics, HBNI, 1/AF Bidhannagar, Salt Lake, Sector-1, Kolkata 700064, India ${ }^{34}$ Inst. for Nucl. Research and Nucl. Energy, Bulgarian Academy of Sciences, BG-1784 Sofia, Bulgaria ${ }^{35}$ Japanese MAGIC Group: Department of Physics, Yamagata University, Yamagata 990-8560, Japan ${ }^{36}$ Finnish MAGIC Group: Astronomy Research Unit, University of Oulu, FI-90014 Oulu, Finland ${ }^{37}$ Croatian MAGIC Group: Ruđer Bošković Institute, 10000 Zagreb, Croatia ${ }^{38}$ INFN MAGIC Group: INFN Sezione di Perugia, I-06123 Perugia, Italy ${ }^{39}$ INFN MAGIC Group: INFN Roma Tor Vergata, I-00133 Roma, Italy ${ }^{40}$ Japanese MAGIC Group: Department of Physics, Konan University, Kobe, Hyogo 658-8501, Japan ${ }^{41}$ also at International Center for Relativistic Astrophysics (ICRA), Rome, Italy ${ }^{42}$ now at Department for Physics and Technology, University of Bergen, NO-5020, Norway ${ }^{43}$ now at University of Innsbruck ${ }^{44}$ also at Port d'Informació Científica (PIC), E-08193 Bellaterra (Barcelona), Spain ${ }^{45}$ now at Ruhr-Universität Bochum, Fakultät für Physik und Astronomie, Astronomisches Institut (AIRUB), 44801 Bochum, Germany ${ }^{46}$ now at Department of Astronomy, University of California Berkeley, Berkeley CA $94720{ }^{47}$ also at Dipartimento di Fisica, Università di Trieste, I-34127 Trieste, Italy ${ }^{49}$ now at Laboratoire d'Annecy de Physique des Particules (LAPP), CNRS-IN2P3, 74941 Annecy Cedex, France ${ }^{50}$ also at INAF Trieste and Dept. of Physics and Astronomy, University of Bologna, Bologna, Italy ${ }^{51}$ ETH Zurich, Institute for Particle Physics and Astrophysics, Otto-Stern-Weg 5, 8093 Zurich, Switzerland 52 TU Dortmund, Experimental Physics 5, Otto-Hahn-Str. 4a, 44227 Dortmund, Germany ${ }^{53}$ University of Geneva, Department of Astronomy, Chemin dÉcogia 16, 1290 Versoix, Switzerland ${ }^{54}$ University of Würzburg, Institute for Theoretical Physics and Astrophysics, Emil-Fischer-Str. 31, 97074 Würzburg, Germany ${ }^{a}$ RWTH Aachen University, D-52074 Aachen, Germany ${ }^{m}$ also in MAGIC ${ }^{55}$ Laboratoire Leprince-Ringuet, École Polytechnique, CNRS/IN2P3, F-91128 Palaiseau, France ${ }^{57}$ INAF - Istituto di Radioastronomia, Bologna, Italy ${ }^{58}$ Space Science Data Center (SSDC) - ASI, via del Politecnico, s.n.c., I-00133, Roma, Italy ${ }^{59} \mathrm{INAF}$-Osservatorio Astronomico di Roma, via di Frascati 33, I-00040 Monteporzio, Italy ${ }^{60}$ Italian Space Agency, ASI, via del Politecnico snc, 00133 Roma, Italy ${ }^{61}$ Department of Physics, University of Colorado Denver, Denver, Colorado, CO 80217-3364, USA ${ }^{62}$ Department of Physics and Astronomy, Brigham Young University, Provo, UT 84602, USA ${ }^{63}$ Institute for Astrophysical Research, Boston University, 725 Commonwealth Avenue, Boston, MA 02215, USA ${ }^{64}$ Astronomical Institute, St.Petersburg State University, Universitetskij Pr. 28, Petrodvorets, 198504 St.Petersburg, Russia ${ }^{65}$ Aalto University Metsähovi Radio Observatory, Metsähovintie 114, FIN-02540 Kylmälä, Finland ${ }^{66}$ Aalto University Department of Electronics and Nanoengineering, P.O. Box 15500, FIN-00076 Aalto, Finland ${ }^{67}$ Astronomy Department, Universidad de Concepción, Casilla 160-C, Concepción, Chile ${ }^{68}$ European Space Agency, European Space Astronomy Centre, C/ Bajo el Castillo s/n, 28692 Villanueva de la Cañada, Madrid, Spain ${ }^{69}$ INAF Istituto di Radioastronomia, Stazione di Medicina, via Fiorentina 3513, I-40059 Villafontana (BO), Italy ${ }^{70}$ Astrophysics Science Division, NASA Goddard Space Flight Center, 8800 Greenbelt Road, Greenbelt, MD 20771, USA ${ }^{71}$ Department of Physics, University of Maryland, Baltimore County, 1000 Hilltop Circle, Baltimore, MD 21250, USA 